\title{
Elevated levels of renal and circulating Nop-7-associated 2 (NSA2) in rat and mouse models of diabetes, in mesangial cells in vitro and in patients with diabetic nephropathy
}

\author{
R. Shahni • L. Gnudi • A. King • P. Jones • A. N. Malik
}

Received: 7 July 2011 / Accepted: 18 October 2011 / Published online: 18 November 2011

(C) Springer-Verlag 2011

\begin{abstract}
Aims/hypothesis We previously found that Nop-7-associated 2 (NSA2), which is involved in ribosomal biogenesis in yeast and is a putative cell cycle regulator in mammalian cells, is elevated in the kidney of Goto-Kakizaki (GK) rat, a spontaneous model of type 2 diabetes. Here we tested the hypothesis that elevated NSA2 is involved in diabetic nephropathy (DN).

Methods We examined Nsa2/NSA2 expression and NSA2 production in two rodent models of diabetes, in cultured renal glomerular cells, and in diabetic patients with and without nephropathy. Patients with nephropathy who had a history of albuminuria were further divided as responders (DN-NA; DN patients normoalbuminuric at the time of this study with a history of albuminuria) and non-responders (DN-A; diabetic nephropathy patients with albuminuria) to current treatment for albuminuria.

Results Renal Nsa2/NSA2 mRNA increased in tandem with hyperglycaemia in GK rats, in a streptozotocin-induced mouse model of diabetes, and in human mesangial cells (HMCs) grown in high glucose $(p<0.05)$. In the mouse model of diabetes, hyperglycaemia resulted in increased
\end{abstract}

Electronic supplementary material The online version of this article (doi:10.1007/s00125-011-2373-4) contains peer-reviewed but unedited supplementary material, which is available to authorised users.

R. Shahni $\cdot$ A. King $\cdot$ P. Jones $\cdot$ A. N. Malik $(\bowtie)$

Diabetes Research Group, Division of Diabetes and Nutritional

Sciences, School of Medicine, Kings College London,

Hodgkin Building, London Bridge,

London SE1 1UL, UK

e-mail: afshan.malik@kcl.ac.uk

L. Gnudi

Cardiovascular Division, School of Medicine,

Kings College London,

London, UK
Nsa2 expression and NSA2 levels in tubular and glomerular cells and in circulating cells; this increase was normalised by diabetes treatment. Circulating NSA2 mRNA levels were elevated in patients with DN independently of body weight (BMI), glycaemic $\left(\mathrm{HbA}_{1 \mathrm{c}}\right)$ and haemodynamic (blood pressure) control, and showed an inverse correlation with renal function (GFR, $p<0.05)$. NSA2 levels were the only variable that showed a significant difference between patients with albuminuria (DN-A) compared with nonalbuminuric patients (DN-NA) and diabetic controls $(p<$ 0.05 ), this increase being independent of all other variables, including GFR.

Conclusion We show for the first time that renal and circulating NSA2/NSA2 levels are increased in hyperglycaemia in experimental models of diabetes, and that circulating $N S A 2$ is elevated in DN patients with albuminuria. Further studies will be required to assess whether NSA2 plays a role in the pathogenesis of $\mathrm{DN}$.

Keywords Diabetic nephropathy · Hyperglycaemia .

Nop-7-associated-2 NSA2

$\begin{array}{ll}\text { Abbreviations } \\ \text { ACR } & \text { Albumin/creatinine ratio } \\ \text { AER } & \text { Albumin excretion rate } \\ \text { CTGF } & \begin{array}{l}\text { Connective tissue growth factor } \\ \text { DN }\end{array} \\ \text { Diabetic nephropathy } \\ \text { DN-A } & \begin{array}{l}\text { Diabetic nephropathy patients with } \\ \text { albuminuria (poor responders) }\end{array} \\ \text { DN-NA } & \begin{array}{l}\text { Diabetic nephropathy patients normoalbuminuric } \\ \text { at the time of this study with a history } \\ \text { of albuminuria (good responders) }\end{array} \\ \text { eGFR } & \begin{array}{l}\text { Estimated GFR } \\ \text { GK }\end{array} \\ \text { HCL1 } 1 & \text { Hairy cell leukaemia protein 1 }\end{array}$




$\begin{array}{ll}\text { HG } & \text { High glucose treatment }(25 \mathrm{mmol} / \mathrm{l}) \\ \text { HMC } & \text { Human mesangial cells } \\ \text { HMCL } & \text { Human mesangial cell line } \\ \text { NA } & \text { Normoalbuminuria } \\ \text { NG } & \text { Normal glucose treatment }(5 \mathrm{mmol} / \mathrm{l}) \\ \text { NSA2 } & \text { Nop-7-associated } 2 \\ \text { qPCR } & \text { Quantitative PCR } \\ \text { RAS } & \text { Renin-angiotensin system } \\ \text { TGF- } \beta 1 & \text { Transforming growth factor beta } 1 \\ \text { UAE } & \text { Urinary albumin excretion }\end{array}$

\section{Introduction}

Diabetic nephropathy (DN) is a chronic kidney disease affecting more than $30 \%$ of patients with diabetes mellitus and is the main cause of end-stage renal disease in the western world. DN develops within 10-30 years after diagnosis of diabetes and is one of the most severe complications of both type 1 and type 2 diabetes [1]. Apart from declining GFR and urinary albumin excretion (UAE), there are no other accepted diagnostic/predictive markers for the detection of DN [2], making it difficult to predict which patients will develop the disease, as urinary albumin can only be detected after a clinically silent phase of 10-20 years of renal damage. Despite the use of therapeutic strategies, a significant percentage of patients that develop albuminuria go on to develop proteinuria and then to endstage renal failure, requiring renal transplantation [3].

The role of hyperglycaemia as a major contributory factor in the development of DN is widely accepted. Clinical randomised studies have shown that early intensive glycaemic control significantly reduces the risk of DN whereas prolonged hyperglycaemia can significantly increase the long term risk of diabetic complications [4-6]. Hyperglycaemia activates several overlapping biochemical pathways resulting in the activation of the polyol pathway, accumulation of AGEs, activation of the hexosamine pathway, activation of the transforming growth factor beta 1 (TGF- $\beta 1$ ) pathway and increased production of reactive oxygen species (ROS system) with resulting oxidative stress, as well as alterations in the renin-angiotensin system (RAS) and the nitric oxide (NO) system [7]. The exact mechanisms by which hyperglycaemia leads to cell and tissue injury are therefore complex, and not fully understood [8].

Differential gene expression has been used by us and others to identify hyperglycaemia-induced renal genes using in vitro and in vivo models and patient samples [9-12]. We previously described the isolation of candidate DN genes that are induced in response to hyperglycaemia and which could play a role in renal damage in DN [13-15].
$C d k 105$, one of the candidate genes that we isolated from the kidneys of a rat model for diabetes, is identical to Nsa2, which encodes Nop-7-associated 2 (NSA2), a protein involved in ribosome biogenesis in yeast [16]. Yeast NSA2 has been described as a nucleolar protein and contains several nuclear localisation signals [16-18]. The human homologue of NSA2 has been shown to regulate the progression of the cell cycle from $\mathrm{G} 1 / \mathrm{S}$, was identified as a tumour suppressor gene described as hairy cell leukaemia gene 1 (HCL1) and may be induced by the cytokine TGF- $\beta 1$ $[19,20]$. There have been no reports to date linking NSA2 to diabetes.

As NSA2 appears to be involved in cell cycle regulation [20], and as we had found the gene by differential screening of kidneys from a diabetic rat model, we tested the hypothesis that renal $N s a 2$ may be induced by hyperglycaemia in vivo in two rodent models of diabetes, the Goto-Kakizaki (GK) rat, a spontaneous model of diabetes [21], and the streptozotocin-induced mouse [22]. The effect of treatment for hyperglycaemia on glucose induction of renal and circulating Nsa2 mRNA in the streptozotocininduced mice was examined. We also used cultured human mesangial cells (HMCs) to test the effect of high glucose on NSA2 mRNA and NSA2 protein levels. We examined circulating Nsa2 mRNA levels to determine whether they reflected the changes seen in the kidneys of the diabetic mouse models. Finally, we measured circulating NSA2 mRNA levels in patients with both type 1 and type 2 diabetes to see if NSA2 levels were associated with DN.

\section{Methods}

\section{Biological materials}

The GK rat and control Wistar rat kidneys (Institute of Nephrology, University of Wales College of Medicine, Cardiff, UK) used in this study have been previously described [13-15]. GK rats are normoglycaemic at 6 weeks $(6 \mathrm{mmol} / \mathrm{l})$, have slightly raised blood glucose at 16 weeks (7-8 $\mathrm{mmol} / \mathrm{l})$ and by 26 weeks are hyperglycaemic (13 mmol/l) [21]. Eight week-old male C57/BL6 mice (King's College London, UK) were rendered diabetic with an injection of $180 \mathrm{mg} / \mathrm{kg}$ streptozotocin (SigmaAldrich, St Louis, USA). Blood glucose concentrations were determined using an Accu-Chek glucose meter (Roche, Burgess Hill, UK). Diabetic mice (blood glucose $>20 \mathrm{mmol} / \mathrm{l}$ ) were used to obtain kidneys and/or blood after either 7 or 30 days from the induction of diabetes and were compared with age-matched non-diabetic mice as controls (blood glucose $<11 \mathrm{mmol} / \mathrm{l}$ ). To treat the diabetes, islets were isolated from pancreases of $8-10$-week-old male C57/BL6 mice using collagenase digestion followed by gradient purification, as 
described previously [22]. Five days after streptozotocin injection, diabetic mice received a suboptimal islet graft of 150 islets transplanted under the left kidney [22]. All animal procedures were conducted in accordance with the UK Home Office Animals (Scientific Procedures) Act 1986.

Primary HMCs (Biowhittaker, Cologne, Germany) and a mesangial cell line (HMCL) [13] were cultured in normal glucose (NG; $5 \mathrm{mmol} / \mathrm{l}$ glucose), high glucose (HG; $25 \mathrm{mmol} / \mathrm{l}$ glucose) and mannitol (NGM; $5 \mathrm{mmol} / 1$ glucose $+20 \mathrm{mmol} / \mathrm{l}$ mannitol) as described previously [22]

Patients with diabetes were recruited with written informed consent from Guy's and St Thomas' hospital clinics under ethical approval from the regional Research Ethics Committee (REC; ref number 07/H0806/120). A random blood glucose level of $\geq 11.1$ or a fasting blood glucose level of $\geq 7 \mathrm{mmol} / \mathrm{l}$ was considered to be indicative of diabetes.

Type 1 diabetes and type 2 diabetes were defined as follows: type 1 diabetes, onset before age 35, insulin therapy within 6 months of diagnosis and no breaks in insulin therapy $>6$ months; type 2 diabetes, onset after age 35 , controlled by diet or established oral hypoglycaemic treatment and/or insulin. Normoalbuminuria was defined as ACR $<2.5 \mathrm{mg} / \mathrm{mmol}$ for men (albumin excretion rate [AER] $<25 \mathrm{mg} /$ day) and ACR $<3.5 \mathrm{mg} / \mathrm{mmol}$ for women (AER $<35 \mathrm{mg} /$ day), albuminuria was defined as ACR $>2.5 \mathrm{mg} / \mathrm{mmol}$ for men (AER $>25 \mathrm{mg} /$ day) and $>3.5 \mathrm{mg} / \mathrm{mmol}$ for women (AER $>35 \mathrm{mg} /$ day). GFR was assessed using the Modification of Diet in Renal Disease (MDRD) formula [23]. For controls without nephropathy, we used patients with type 1 diabetes and type 2 diabetes with $\geq 20$ or $\geq 10$ years of diabetes duration, respectively, without history of albuminuria, with normal renal function and normal blood pressure $(\leq 130 / 80 \mathrm{mmHg})$ and taking no antihypertensive agents. The study adhered to the Ethical Principles for Medical Research Involving Human Subjects, World Medical Association Declaration of Helsinki. Whole blood was obtained from the patients $(0.2 \mathrm{ml})$ and immediately placed in RNA later $(200 \mu \mathrm{l})$. The blood samples were frozen within $2 \mathrm{~h}$ of collection at $-20^{\circ} \mathrm{C}$.

\section{RNA extraction and real-time PCR}

Total RNA was extracted from whole peripheral blood using RNAeasy blood kit (ABI, Forster City, CA, USA) and tissues using RNAqueous-4PCR kit (ABI) and was reverse transcribed to cDNA using the high capacity RNA to cDNA kit (ABI). Oligonucleotide primers (electronic supplementary material [ESM] Table 1) were designed using the universal probe library (Roche), and synthesised (Sigma-Aldrich). Real-time quantitative PCR (qPCR) was carried out using SYBR green (Qiagen, Crawley, UK) [13-15] in the Roche LC480 Light Cycler. Copy number values were expressed as relative to $G A P D H$. All reactions were performed in triplicate in the presence of calibration standards containing a dilution series to generate quantification data. Northern blotting was carried out as previously described [13].

\section{NSA2 protein detection}

An NSA2 polyclonal antibody was custom generated using a 15 residue peptide sequence (GFTRKPPKYERFIR) in New Zealand White rabbits (GenScript, Piscataway, NJ, USA). A tubulin (TU-02) antibody (Santa Cruz Biotechnology) was used as an equal loading control. The secondary antibody was horseradish peroxidase (HRP) (western blot) and FITCconjugated (immunofluorescent) anti-rabbit (Santa Cruz, CA, USA). The NSA2 antibody was tested against the peptide used to produce it using western blotting to check specificity. The negative control had no primary, and only secondary, antibody. The kidney sections were stained blue with DAPI and viewed under a fluorescent microscope (Eclipse TE 2000U; Nikon, Oxford, UK).

\section{Image J analysis}

ImageJ (v3.91, http://rsb.info.nih.gov/ij) was used to measure fluorescence intensity ( $n=3$ for each condition) and band intensity in western blots.

\section{Statistical analysis}

Using SPSS 17, independent sample $t$ tests, ANOVA/ bivariate correlations, and binary logistic regression were used. Data were expressed as either median or mean $\pm \mathrm{SD}$. Differences were considered significant with $p$ values $<0.05$ and highly significant with $p$ values $<0.005$.

\section{Results}

We previously cloned a number of genes from kidneys of the GK rat, a model of type 2 diabetes, on the basis of their elevated renal expression in association with hyperglycaemia [10, 11]. Cdk105 (accession number NM_014886) was one of the clones we isolated by differential screening [15], which represented an abundantly expressed renal mRNA and contained a $1.1 \mathrm{~kb}$ insert with a nuclear localisation signal. The human homologues of rat Nsa2 were identified using blast as the hairy cell leukaemia gene HCL1 [19] and TINP1, the latter encoding a putative TGF- $\beta 1$-induced protein (accession number $\mathrm{NC}$ 007868). CDK105 and HCL1/TINP1 subsequently turned out to be homologues 
of yeast $N s a 2$ [16]; therefore the human version of this gene will be referred to from here onwards as NSA2.

Hyperglycaemia results in increased renal Nsa2/NSA2 levels in vivo and in vitro

Renal levels of Nsa2 in the GK rat, a spontaneous model of diabetes To test the effect of hyperglycaemia on renal $\mathrm{Nsa} 2$ mRNA levels, we compared Nsa2 expression in the kidneys of a spontaneous model of mild diabetes, the GK rat, with Wistar rats, from which the GK rat is derived, as controls. The GK rats were normoglycaemic at 6 weeks $(<6 \mathrm{mmol} / \mathrm{l})$ and progressively developed hyperglycaemia, being hyperglycaemic by 26 weeks (glucose level $>13 \mathrm{mmol} / \mathrm{l}$ ), whereas the Wistar rats remained normoglycaemic at all ages $(<6 \mathrm{mmol} / \mathrm{l})$ [11]. Renal Nsa2 mRNA levels increased in association with hyperglycaemia in the GK rat, showing a significant increase at 26 weeks compared with 6 weeks (Fig. 1). There was no change in Nsa2 mRNA levels in agematched Wistar rat kidneys.

Renal levels of Nsa2 in streptozotocin-induced diabetic mice A mouse streptozotocin-induced model of diabetes was used to determine whether we could confirm the elevated renal expression of $N s a 2$ in response to hyperglycaemia in a different model from the GK rat. C57/BL6 mice were rendered diabetic by administration of streptozotocin and maintained in the diabetic state (blood glucose $>20 \mathrm{mmol} / \mathrm{l}$ ) for 4 weeks, after which renal levels of $\mathrm{Nsa2}$ were compared between diabetic (blood glucose $>20 \mathrm{mmol} / \mathrm{l}$ ) and control (blood glucose $<11 \mathrm{mmol} / \mathrm{l}$ ) mice. Nsa2 mRNA was elevated by $\sim 2.3$-fold in kidneys from the diabetic mouse model (Fig. 2a, $p<0.005$ ). Protein levels of NSA2 were also elevated in diabetic kidneys with the $30 \mathrm{kDa}$ NSA2 band clearly increased in diabetic vs control kidneys (Fig. 2b). Immunohistochemistry revealed abundant levels of

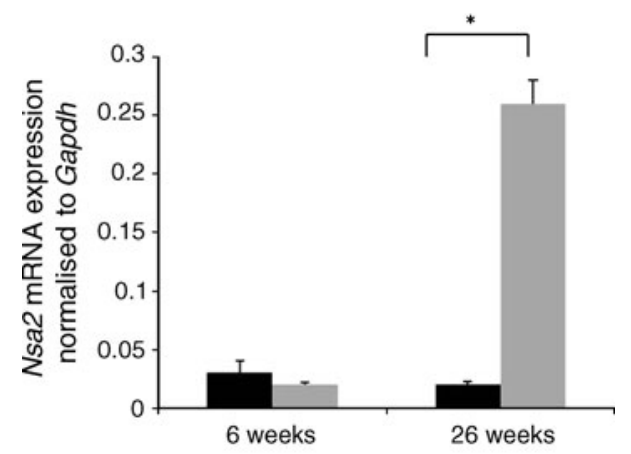

Fig. 1 Rat $N s a 2$ mRNA levels are elevated in diabetic GK rat kidney. Real-time qPCR data showing Nsa2 mRNA and Gapdh control in control Wistar and the diabetic GK rat at different stages. Renal Nsa2 levels increased in the diabetic kidney (26-week-old GK rat) whereas they remained relatively constant in control Wistar rats. Black bars, control rats, grey bars, GK rats. ${ }^{*} p<0.05$
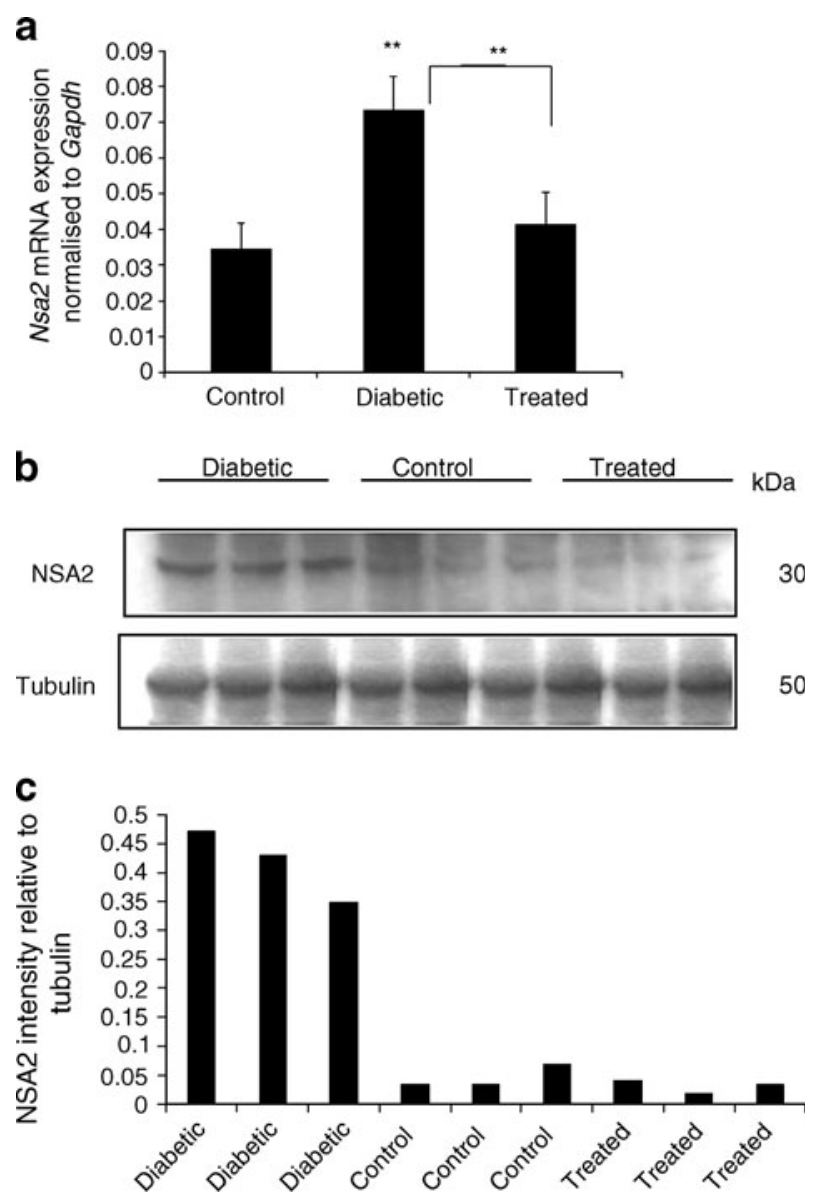

Fig. 2 Renal Nsa2 mRNA and NSA2 protein levels in control, diabetic and treated mice. a Total kidney RNA from control (blood glucose $<11 \mathrm{mmol} / \mathrm{l}$ ), streptozotocin-induced diabetic mice (blood glucose $>20 \mathrm{mmol} / 1$ maintained for 4 weeks) and islet-transplantationtreated mice (blood glucose $<11.1 \mathrm{mmol} / \mathrm{l}$ maintained post transplant for 4 weeks) $(n=3)$ was used to determine Nsa2 mRNA copy numbers relative to Gapdh mRNA using real-time $\mathrm{qPCR} .{ }^{* *} p<0.005$ vs control. b Equal amounts of mouse kidney tissue lysate protein $(25 \mu \mathrm{g})$ were subjected to SDS-PAGE and the quantity of NSA2 protein was detected by immunoblot analysis using rabbit polyclonal IgG primary antibody raised against NSA2 (top panel). The blot was stripped and re-probed with anti-tubulin antibody (bottom panel) to demonstrate equal loading of protein in all lanes $(n=3)$. $\mathbf{c}$ Protein bands quantified by densitometry. Each bar represents the intensity of NSA2 relative to tubulin

NSA2 protein throughout the mouse kidney. Kidneys from diabetic mice showed tubular and glomerular hypertrophy and an increase of greater than fivefold in NSA2 staining compared with the controls (Fig. 3a, b), as well as nuclear staining in the glomeruli (Fig. 3c). These data show that NSA2 is produced throughout the kidney and support the view that hyperglycaemia results in increased renal Nsa2/ NSA2 levels.

Effect of high glucose on NSA2 levels in cultured glomerular cells As we had shown that hyperglycaemia resulted in increased renal Nsa2 mRNA in vivo in both the 
a

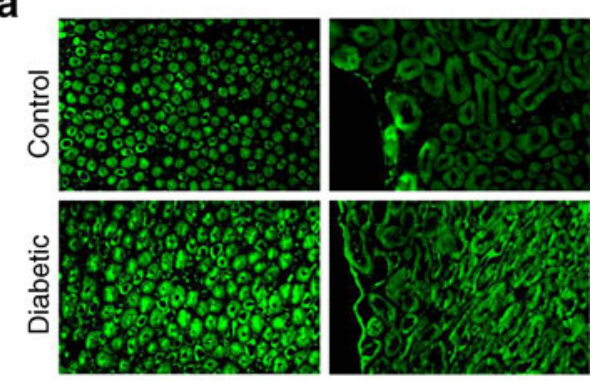

b

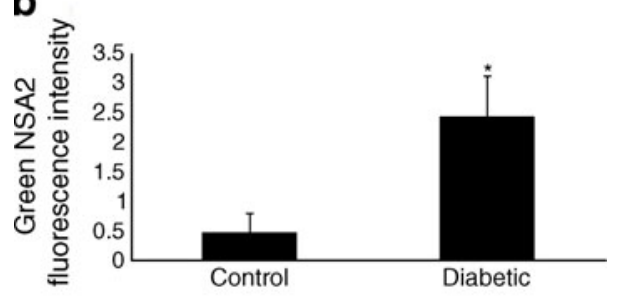

C

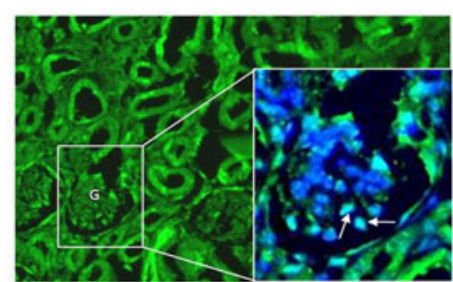

Fig. 3 NSA2 protein in control and diabetic mouse kidney. Sections of kidneys from control mice (blood glucose $<11 \mathrm{mmol} / \mathrm{l}$ ) and streptozotocin-induced diabetic mice (blood glucose $>20 \mathrm{mmol} / 1$ maintained for 30 days) were labelled with the NSA2 primary antibody and FITC-conjugated secondary antibody. a Sections of control and diabetic kidneys showing NSA2 (green) present throughout the kidney and higher levels of NSA2 in kidneys from streptozotocin-treated mice compared with the control; magnification $\times 200$. b Fluorescence intensity of at least three separate high power field images, measured using ImageJ, of diabetic mouse kidney vs control ${ }^{*} p<0.05$. c A section from a diabetic mouse model kidney showing NSA2 (green) in tubular and glomerular cells. The nuclei are stained with DAPI (blue). One glomerulus has been enlarged to show the nuclear location of NSA2 protein in diabetic kidney (light blue); magnification $\times 400$

GK rat and streptozotocin-induced mice, we examined cultured human renal cells to determine whether they showed a response to increased glucose in vitro. Primary HMCs were cultured in triplicate in normal glucose ( $\mathrm{NG} ; 5 \mathrm{mmol} / \mathrm{l}$ glucose) and high glucose (HG; $25 \mathrm{mmol} / \mathrm{l}$ glucose). A mannitol control (NGM; $5 \mathrm{mmol} / \mathrm{l}$ glucose $+20 \mathrm{mmol} / \mathrm{l}$ mannitol) was included to test for any osmolarity effect. As there is no universal housekeeping gene, we measured the expression of $B 2 M, \beta$-actin and $G A P D H$ in our experimental condition and used geNorm (http://medgen.ugent.be/ jvdesomp/genorm/) to calculate the most stable gene. $G A P D H$ was found to be most stably expressed in mesangial cells. NSA2 mRNA copy numbers relative to $G A P D H$ were determined using real-time qPCR and NSA2 protein was measured using western blotting (Fig. 4a, b). Using a post hoc Tukey's test for
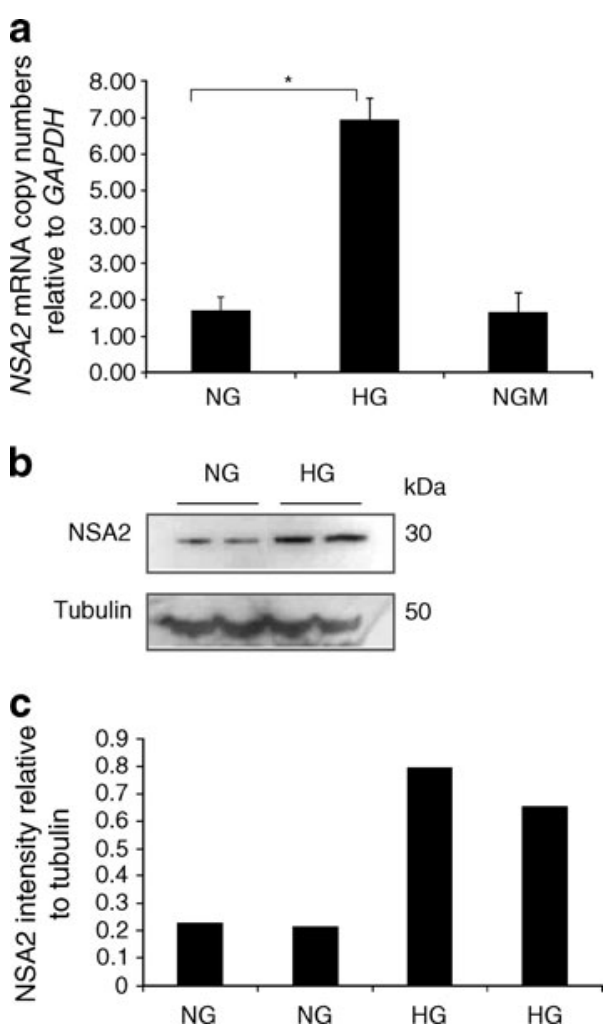

Fig. 4 The effect of high glucose on NSA2 mRNA and NSA2 protein in cultured HMCs. After being synchronised, HMCs were incubated in $5 \mathrm{mmol} / \mathrm{l}$ glucose (NG), $25 \mathrm{mmol} / \mathrm{l}$ glucose (HG) or $5 \mathrm{mmol} / \mathrm{l}$ glucose + $20 \mathrm{mmol} / 1$ mannitol (NGM) for 3 days. a NSA2 mRNA copy numbers relative to $G A P D H$ were determined using real-time qPCR $(n=3)$; ${ }^{*} p<0.05$. b Equal quantities of cell lysate protein $(25 \mu \mathrm{g})$ were subjected to SDS-PAGE and the amount of NSA2 protein was detected by immunoblot analysis using rabbit polyclonal IgG primary antibody raised against NSA2 (top panel). The blot was stripped and re-probed with anti-tubulin antibody (bottom panel) to demonstrate equal loading of protein in all lanes. c Protein bands quantified by densitometry. The bars represent intensity of NSA2 relative to tubulin

analysis of variance, we found no significant difference between NG and NGM ( $p>0.05)$, showing that there is no osmolarity effect in play. The cells grown in $\mathrm{HG}(6.96 \pm 0.56)$ show a significant fourfold increase in NSA2 mRNA copy numbers compared with NG $(1.73 \pm 0.34)$ or NGM $(p<0.05)$. The increases in NSA2 mRNA were accompanied by increased NSA2 protein (Fig. $4 b, c)$. Similar results were obtained with a transformed mesangial cell line (HMCL). HMCLs grown in HG showed a fivefold increase compared with cells grown in NG $(p<0.05$; data not shown). These data show that HMCs express $N S A 2$ and that the human NSA2 gene is upregulated by high glucose in cultured mesangial cells.

Treatment of diabetes normalises hyperglycaemia-induced renal NSA2

To test whether hyperglycaemia is the cause of $\mathrm{Nsa2}$ induction in the diabetic kidney, streptozotocin-induced 
diabetic mice were treated using islet transplantation. C57/ BL6 mice were rendered diabetic by administration of streptozotocin and maintained in the diabetic state (blood glucose $>20 \mathrm{mmol} / \mathrm{l}$ ) for 5 days, after which they were treated with islet transplantation, and maintained for 4 weeks (blood glucose $<11.1 \mathrm{mmol} / \mathrm{l}$ ). Islet transplantation was used in preference to insulin treatment of diabetes as the transplanted islets provide a more immediate minute by minute physiological response to blood glucose fluctuations, whereas with insulin injections there is a bigger fluctuation in blood glucose. Renal Nsa2 mRNA in treated mice $(0.041 \pm 0.008)$ showed no significant difference from the control $(0.035 \pm 0.007)$ but was significantly lower than in the diabetic mice $(0.073 \pm 0.008, p<0.05$, Fig. 2$)$. These data suggest that increased $\mathrm{Nsa2}$ levels in response to hyperglycaemia can be corrected by treatment for diabetes, and further confirm the hypothesis that hyperglycaemia results in increased renal Nsa2/NSA2 levels.

Hyperglycaemia results in increased NSA2 in peripheral blood in mice

Having established that glucose/hyperglycaemia can enhance renal Nsa2 mRNA/NSA2 protein in vivo and in vitro, we wanted to determine whether glucose-induced changes in Nsa2 expression seen in renal cells are also accompanied by changes in Nsa2 mRNA levels in circulating cells. Peripheral blood from control and diabetic mice (whole blood) was used to measure Nsa2 mRNA levels. Nsa2 mRNA was elevated 2.5-fold in blood from the diabetic mouse models compared with control mouse blood $(p<0.005$; Fig. 5). Treatment of diabetes resulted in normalisation of renal Nsa2 mRNA levels. Mice that had been in the diabetic state for 4 weeks, then treated with islet transplantation and maintained for 7 days at normal glucose levels, showed no significant difference from controls $(p>$

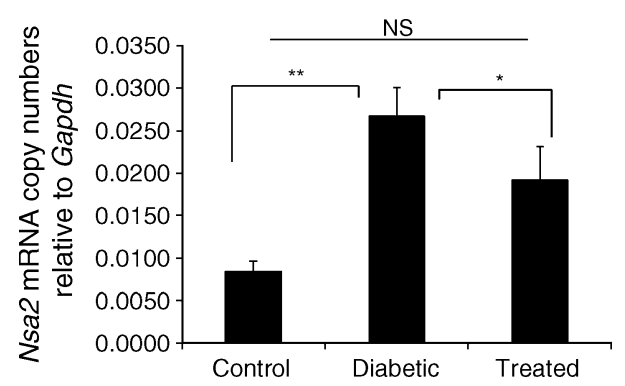

Fig. 5 Nsa2 mRNA levels in peripheral blood of control (blood glucose $<11 \mathrm{mmol} / \mathrm{l}$ ), streptozotocin-induced diabetic model (blood glucose $>20 \mathrm{mmol} / \mathrm{l}$ maintained for 4 weeks) and islet-transplantationtreated (blood glucose $<11.1 \mathrm{mmol} / 1$ maintained post transplant for 7 days) mice. Total RNA from whole blood was used to determine $N S A 2$ mRNA levels using real-time qPCR $(n=3)$. The mean values of three different experiments are shown. Values shown are copy numbers of Nsa2 mRNA relative to Gapdh; ${ }^{*} p<0.05,{ }^{* *} p<0.005$
$0.05)$, suggesting that Nsa2 mRNA levels had returned to normal (Fig. 5).

Increased circulating NSA2 mRNA in patients with DN

Circulating NSA2 mRNA levels were measured in diabetic patients selected consecutively and analysed in the following groups:

1. Diabetic controls: patients with long duration of diabetes ( $>20$ years for type 1 diabetes, $>10$ years for type 2 diabetes), with no history of albuminuria and with normal renal function $(n=17)$;

2. DN patients: patients with current or history of albuminuria $(n=51)$.

The baseline characteristics of these two groups are shown in Table 1. The DN patients showed decreased GFR, and increased albumin to creatinine ratio (ACR), BMI and random blood glucose $(p<0.05)$ compared with diabetic controls. There was no significant difference between the $\mathrm{DN}$ and control groups in terms of $\mathrm{HbA}_{1 \mathrm{c}}$, blood pressure, cholesterol, sex or duration of diabetes. The DN patients had $\sim 3.5$-fold higher circulating NSA2 mRNA $(p<0.05)$ compared with controls.

Binary logistic regression analysis was performed with nephropathy as the dependent variable and $\log _{\mathrm{e}} N S A 2$ mRNA copy numbers, actual values for age, sex, BMI, $\mathrm{HbA}_{1 \mathrm{c}}$, diastolic and systolic BP, and diabetes duration as predictor variables (Table 2). $\log _{\mathrm{e}}$ NSA2 copy numbers showed a significant and positive association with nephropathy, and a negative association with GFR, but not with any of the other variables. Each unit increase in $\log _{\mathrm{e}} N S A 2$ mRNA copy numbers score was associated with an increase in the odds for DN by a factor of 3.8.

Increased NSA2 mRNA in patients with microalbuminuria/ proteinuria (poor responders) compared

with normoalbuminuric patients (good responders)

Nephropathy patients with a history of albuminuria in this study were further divided into two groups:

1. Patients with a history of DN but who were normoalbuminuric at the time of this study (DN-NA, $n=21$ );

2. Patients with $\mathrm{DN}$ with current micro/macroalbuminuria (DN-A, $n=30$; Table 1).

Diabetes and hypertension treatments for the DN group were according to guidelines and similar for both the DN-A and the DN-NA groups. The DN-NA group were normoalbuminuric and therefore were defined as good responders whereas the DN-A group showed residual albuminuria despite optimal therapy with RAS inhibitors, i.e. were defined as poor responders to treatment. Compared with 
Table 1 Baseline characteristics of diabetic patients with and without nephropathy and according to renal function (means \pm SD)

\begin{tabular}{|c|c|c|c|c|}
\hline Variable & Diabetic controls $(n=17)$ & DN (full set) $(n=51)$ & DN-NA $(n=21)$ & DN-A $(n=30)$ \\
\hline Age (years) & $50 \pm 15$ & $61 \pm 13$ & $62 \pm 14$ & $60 \pm 13$ \\
\hline Sex (male/female) & $8: 9$ & $27: 24$ & $9: 12$ & $19: 11$ \\
\hline BMI $\left(\mathrm{kg} / \mathrm{m}^{2}\right)$ & $26 \pm 4$ & $30 \pm 6^{*}$ & $28 \pm 5^{*}$ & $30 \pm 6^{*}$ \\
\hline Mean BP (mmHg) & $87 \pm 6$ & $91 \pm 10$ & $86 \pm 10$ & $94 \pm 8^{\dagger \dagger}$ \\
\hline $\mathrm{HbA}_{1 \mathrm{c}}(\%)$ & $8 \pm 1.5$ & $7.6 \pm 1.3$ & $7.4 \pm 1.4$ & $7.7 \pm 1.3$ \\
\hline $\mathrm{HbA}_{1 \mathrm{c}}(\mathrm{mmol} / \mathrm{mol})$ & 64 & 59 & 59 & 64 \\
\hline Blood glucose (mmol/l) & $11.5 \pm 4.8$ & $20.5 \pm 4^{*}$ & $9.9 \pm 3.4^{*}$ & $10.2 \pm 4.4^{*}$ \\
\hline $\mathrm{SCr}(\mu \mathrm{mol} / \mathrm{l})$ & $63 \pm 14$ & $122 \pm 79 * *$ & $100 \pm 44 * *$ & $140 \pm 96^{* * \dagger}$ \\
\hline Albuminuria (mg/day) & $4.6 \pm 3.7$ & $113 \pm 170^{* *}$ & $11.9 \pm 20^{* *}$ & $196 \pm 193 * * \dagger$ \\
\hline $\operatorname{ACR}(\mathrm{mg} / \mathrm{mmol})$ & $0.9 \pm 0.7$ & $17 \pm 46^{* *}$ & $0.7 \pm 0.5^{* *}$ & $31 \pm 59 * * \dagger$ \\
\hline eGFR $\left(\mathrm{ml} \mathrm{min}{ }^{-1} 1.73 \mathrm{~m}^{-2}\right)$ & $104 \pm 18$ & $64 \pm 33 * *$ & $67 \pm 28^{* *}$ & $62 \pm 37 * *$ \\
\hline Cholesterol (mmol/l) & $4.3 \pm 0.7$ & $3.9 \pm 0.8$ & $3.5 \pm 0.7$ & $4.2 \pm 0.8$ \\
\hline Diabetes duration (years) & $22 \pm 10$ & $20 \pm 14$ & $25 \pm 16$ & $18 \pm 12$ \\
\hline NSA 2 copy numbers & $2.1 \pm 4.0$ & $7.5 \pm 11 *$ & $4.1 \pm 7$ & $9.4 \pm 13^{*}$ \\
\hline NSA2 median & 1 & 2.9 & 2.4 & 5.8 \\
\hline
\end{tabular}

Data are means $\pm \mathrm{SD}$, except where otherwise indicated

${ }^{*} p<0.05, * * p<0.005$ compared with controls, ${ }^{\dagger} p<0.05,{ }^{\dagger \dagger} p<0.005$ compared with DN-NA

$\mathrm{SCr}$, serum creatinine. Baseline characteristics of patients according to type of diabetes are shown in ESM Table 3

diabetic controls, patients within both the DN-NA and DNA groups showed decreased GFR and increased ACR ratio, BMI and random blood glucose $(p<0.05)$. There was no significant difference between the groups and controls in terms of $\mathrm{HbA}_{1 \mathrm{c}}$, blood pressure, cholesterol, duration of diabetes, or sex. The DN-A group had significantly higher NSA2 mRNA levels than the diabetic controls $(p<0.05)$, whereas the DN-NA group showed an increase that was not statistically significant (Fig. 6). Binary logistic analysis showed that both nephropathy groups had a positive association with NSA2 and a negative association with GFR compared with controls (Table 2). However, whilst NSA2 levels could distinguish between the two renal groups, GFR levels could not. The DN-NA group showed a positive association with NSA2 mRNA levels and this difference was independent of GFR. Therefore, although GFR can distinguish between DN and controls, NSA2 mRNA can be used to differentiate between the DN-A and the DN-NA groups, i.e. between patients who are good responders and patients who are poor responders to treatment.

\section{Discussion}

We describe evidence for the first time that NSA2, a putative cell cycle regulator [20] needed for yeast ribosomal biogenesis [16], is involved in diabetes and may play a role in hyperglycaemia-induced pathways in the kidney that lead to DN. Furthermore, circulating NSA2 mRNA levels are increased in patients with DN, suggesting that NSA2 might be involved in the pathogenesis of $\mathrm{DN}$.

The function of NSA2 in diabetic kidney or circulating cells is unknown. NSA2 is a highly conserved $30-\mathrm{kDa}$ protein, known to be required for the production of yeast ribosomes and, consequently, for cell proliferation [16]. Depletion or absence of NSA2 causes a decrease in free $60 \mathrm{~S}$ ribosomal subunits and $25 \mathrm{~S}$ and 5.8S ribosomal RNA

Table 2 Summary of binary regression analysis ( $p$ values) showing that NSA2 mRNA levels correlate with severity of nephropathy independently of eGFR

\begin{tabular}{lllll}
\hline Variable & Control vs DN & Control vs DN-NA & Control vs DN-A & DN-NA vs DN-A \\
\hline Log NSA2 & $0.03^{*}$ & $0.046^{*}$ & $0.036^{*}$ & $0.006^{*}$ \\
eGFR & $0.01^{*}$ & $0.018^{*}$ & $0.047^{*}$ & 0.896 \\
\hline
\end{tabular}

Log NSA2 was positively associated and eGFR was negatively associated with nephropathy (DN, DN-A or DN-NA)

${ }^{*} p<0.05$ (full analysis shown in ESM Table 2) 


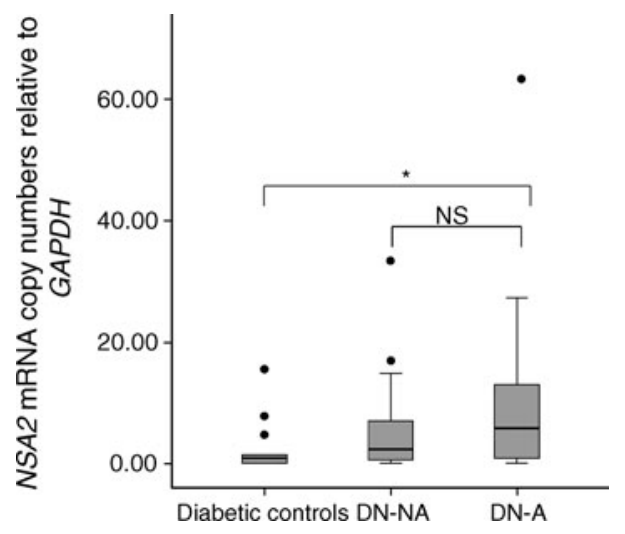

Fig. 6 NSA2 mRNA copy number in peripheral blood from diabetic patients with and without nephropathy according to their renal function. Comparison of patients with $\mathrm{DN}$ who also had albuminuria (DN-A, $n=30)$ and DN patients who had reverted to normoalbuminuria (DN-NA, $n=21)$ with diabetic controls $(n=17)$. NSA2 mRNA values increased in DN-A compared with control patients $\left({ }^{*} p<0.05\right)$. Black dots represent the outliers. NSA2 mRNA copy numbers in patients with and without nephropathy, categorised according to their type of diabetes, are shown in ESM Fig. 1

levels $[16,24]$. When ribosome biogenesis was blocked upstream of NSA2, the protein was largely depleted, suggesting that its cellular levels are tightly regulated [16]. Lebreton et al. (2006) proposed that a pathological overexpression of the normally tightly regulated $\mathrm{Nsa} 2$ gene might favour tumour progression [16]. There have been very few studies on the possible function of NSA2 in humans and other organisms. Human and yeast NSA2 may have similar functions as the two proteins are $99 \%$ identical and human NSA2 can functionally complement yeast $N s a 2$ mutants (YER126C) [16]. Zhang et al. (2010) reported that NSA2 is a nucleolar protein involved in cell proliferation and cell cycle regulation. Overexpression of NSA2 prompted cell growth in different human cell lines and appeared to regulate $\mathrm{G} 1 / \mathrm{S}$ transition in the mammalian cell cycle, as NSA2 knockdown cells were arrested at this stage [20]. The putative role of NSA2 in the cell cycle is supported by an earlier study, which proposed that the gene encoding HCL1, a protein identical to NSA2, is an oncogene [19]. A putative TGF- $\beta 1$-induced protein (TINP1) in a microarray screen (NC_007868) has the same protein sequence as NSA2 and HCL1.

In the current study we found that hyperglycaemia significantly increases renal NAS2 levels in vivo in two different rodent models of diabetes and in vitro in cultured human renal cells. Furthermore, when streptozotocininduced hyperglycaemia was treated with islet transplantation in mice, renal Nsa2 mRNA levels were normalised, supporting the view that hyperglycaemia induces Nsa2. Hyperglycaemia is known to lead to changes in renal cells, affecting many different renal cell types, such as tubular epithelial cells, glomerular mesangial cells, podocytes, interstitial fibroblasts and others [25], and is also a major contributory factor to the development of DN [5]. We found high levels of NSA2 throughout the kidneys of the streptozotocin mouse model, and in particular in tubular cells. It is not clear from our study whether hyperglycaemia directly increases NSA2 or whether the increase is a secondary effect. As yeast NSA2 is required for ribosomal assembly $[16,26]$, there is a possibility that increased NSA2 levels could affect ribosome biogenesis in diabetes. Increased ribosome biogenesis in diabetes has been shown in mice with pancreatic beta cell failure, where several genes involved in ribosomal assembly are upregulated, and in glomerular epithelial cells, where high glucose increases ribosomal biogenesis and leads to renal hypertrophy in rodents models for type 1 and type 2 diabetes [27, 28]. It would be interesting to determine whether increased NSA2 levels lead to increased ribosomal biogenesis and whether NSA2 plays a role in the unfolded protein response and endoplasmic reticulum stress, which has been linked to kidney damage [29].

We found that, in diabetic mice, levels of circulating Nsa2 mRNA increased in response to hyperglycaemia alongside renal Nsa2 mRNA levels, suggesting that increase in NSA2 in response to hyperglycaemia is not kidney specific and that circulating NSA2 is also affected by diabetes. We therefore decided to see whether circulating NSA2 mRNA levels are affected in diabetes patients with and without nephropathy.

We compared the levels of circulating NSA2 mRNA in diabetic patients with and without nephropathy and found that DN patients had $~ 3.5$-fold higher NSA2 mRNA levels $(p<0.05)$ and showed an inverse correlation with estimated GFR (eGFR) but not with other variables, including age, sex, duration of diabetes, $\mathrm{BMI}, \mathrm{HbA}_{1 \mathrm{c}}$ or blood pressure. It is surprising, in the context of our observation that renal NSA2 is increased during hyperglycaemia, that we do not see any effect of metabolic control $\left(\mathrm{HbA}_{1 \mathrm{c}}\right)$ on NSA2 levels. However, as we do not know the function of elevated NSA2 in diabetes and we have not compared levels of NSA2 in healthy controls and patients with diabetes, this observation is an initial finding and needs further confirmation.

The early detection of DN involves measurement of UAE and declining GFR [30]. However, as these methods usually detect renal dysfunction only after a long period of clinical silence, during which significant kidney damage can occur, there is a need to identify new biomarkers that could have predictive power. Emerging candidate biomarkers of renal dysfunction include markers of glomerular damage, oxidative stress and inflammation. To date, UAE remains the universally accepted biomarker in clinical practice [31]. One promising new biomarker is cystatin $\mathrm{C}$, a cysteine protease inhibitor that is increased in serum and urine of patients with DN [32-34]. Cystatin C is increased 
in serum and urine alongside an increasing degree of albuminuria, being higher in macroalbuminuric patients, and correlates with other risk factors, including GFR, sex, $\mathrm{ACR}$ ratio, and $\mathrm{C}$ reactive protein [35]. Another biomarker, urinary liver-type fatty acid-binding protein (L-FABP), was found to be elevated in individuals with type 2 diabetes compared with controls, and correlated with blood pressure, $\mathrm{HbA}_{1 \mathrm{c}}$, eGFR and cholesterol [36]. Connective tissue growth factor (CTGF) another putative DN biomarker, is a profibrotic mediator that is upregulated in tubular epithelial cells by TGF- $\beta 1$ and is proposed to play an important role in renal fibrogenesis [37]. In some studies, CTGF levels show correlation with other DN risk factors, such as GFR, albuminuria, creatinine clearance and duration of diabetes [38].

Our finding that NSA2 mRNA levels can distinguish between the DN-A group compared with the DN-NA group amongst our patient population suggests that elevated levels of NSA 2 might be used to distinguish between patients who are good and poor responders to treatment for $\mathrm{DN}$. However, as this is a retrospective study and we do not have the baseline values (before treatment) for other variables such as $\mathrm{ACR}, \mathrm{HbA}_{1 \mathrm{c}}$ and duration of $\mathrm{DN}$, it is not possible to conclude whether NSA2 plays a mechanistic role in renal impairment.

Our study used real-time qPCR to measure circulating NSA2 mRNA in whole blood. Development of an ELISA assay to allow the accurate quantification of serum and urine NSA2 protein is required to evaluate the potential of NSA2 as a putative biomarker.

In conclusion, we have shown that renal and circulating NSA2 mRNA is upregulated by hyperglycaemia and that NSA 2 mRNA levels associate with renal impairment in our population of patients, independent of other risk markers. These data suggest that NSA2 may play a pathogenetic role in DN. Future studies should evaluate the levels of NSA2 in patients with renal impairment without diabetes. Whether the increase in NSA2 levels is causative or a consequence of the disease could be established in a longitudinal study.

Acknowledgements Thanks to C. Rackham for help with mouse tissue collection and immunohistochemistry, M. Christie for advice on ImageJ analysis, and to C. Morris, Q. Zaidi and M. El-Mahdi, who helped to clone and sequence the original rat CDK105, now named NSA2. Patient sample collection was funded by an innovation award from SEEDA/JJ. Rodent work was partially funded by a Diabetes UK grant.

Contribution statement All authors contributed to the conception and design, or analysis and interpretation of data; drafted the article or revised it critically for important intellectual content; and approved the final version to be published.

Duality of interest The authors declare that there is no duality of interest associated with this manuscript.

\section{References}

1. Schena FB, Gesualdo (2005) Pathogenetic mechanisms of diabetic nephropathy. J Am Soc Nephrol 16:S30-S33

2. Perkins BA, Ficociello LH, Ostrander BE et al (2007) Microalbuminuria and the risk for early progressive renal function decline in type 1 diabetes. J Am Soc Nephrol 18:1353-1361

3. Viberti GC, Bilous RW, Mackintosh D, Bending JJ, Keen H (1983) Long term correction of hyperglycaemia and progression of renal failure in insulin dependent diabetes. BMJ (Clin Res Ed) 286:598-602

4. Genuth S (2006) Insights from the diabetes control and complications trial/epidemiology of diabetes interventions and complications study on the use of intensive glycemic treatment to reduce the risk of complications of type 1 diabetes. Endocr Pract 1:34-41

5. The Diabetes Control and Complications Trial Research Group (1993) The effect of intensive treatment of diabetes on the development and progression of long-term complications in insulin-dependent diabetes mellitus. N Engl J Med 329:977-986

6. UK Prospective Diabetes Study (UKPDS) Group (1998) Intensive blood-glucose control with sulphonylureas or insulin compared with conventional treatment and risk of complications in patients with type 2 diabetes (UKPDS 33). Lancet 352:837-853

7. Brownlee M (2001) Biochemistry and molecular cell biology of diabetic complications. Nature 4:813-820

8. Sheetz MJ, King GL (2002) Molecular understanding of hyperglycemia's adverse effects for diabetic complications. JAMA 27:2579-2588

9. Murphy M, Crean J, Brazil DP et al (2008) Regulation and consequences of differential gene expression in diabetic kidney disease. Biochem Soc Trans 36:941-945

10. Page RA, Morris CA, Williams CJ, Von RC, Malik AN (1997) Isolation of diabetes-associated kidney genes using differential display. Biochem Biophys Res Commun 232:49-53

11. Page RA, Malik AN (2003) Elevated levels of beta defensin-1 mRNA in diabetic kidneys of GK rats. Biochem Biophys Res Commum 310:513-521

12. Cohen CD, Lindenmeyer MT, Eichinger F et al (2008) Improved elucidation of biological processes linked to diabetic nephropathy by single probe-based microarray data analysis. PLoS One 13:e2937

13. Malik AN, Rossios C, Al-Kafaji G, Shah A, Page RA (2007) Glucose regulation of CDK7, a putative thiol related gene, in experimental diabetic nephropathy. Biochem Biophys Res Commun 25:237-244

14. Malik AN, Al-Kafaji G (2007) Glucose regulation of $\beta$-defensin 1 mRNA in human renal cells. Biochem Biophys Res Commun 353:318-323

15. Al-Kafaji G, Malik AN (2010) Hyperglycemia induces elevated expression of thyroid hormone binding protein in vivo in kidney and heart and in vitro in mesangial cells. Biochem Biophys Res Commun 391:1585-1591

16. Lebreton A, Saveanu C, Decourty L, Jacquier A, Fromont-Racine M (2006) NSA2 is an unstable, conserved factor required for the maturation of 27 SB pre-rRNAs. J Biol Chem 281:27099-27108

17. Scherl A, Coute Y, Deon C et al (2002) Functional proteomic analysis of human nucleolus. Mol Biol Cell 13:4100-4109

18. Andersen JS, Lam YW, Leung AK et al (2005) Nucleolar proteome dynamics. Nature 433:77-83

19. Wu X, Ivanova G, Merup M et al (1999) Molecular analysis of the human chromosome 5q13.3 region in patients with hairy cell leukemia and identification of tumor suppressor gene candidates. Genomics 60:161-171

20. Zhang H, Ma X, Shi T, Song Q, Zhao H, Ma D (2010) NSA2, a novel nucleolus protein regulates cell proliferation and cell cycle. Biochem Biophys Res Commun 391:651-658 
21. Goto Y, Kakizaki M (1981) The spontaneous diabetes rat: a model of non-insulin-dependent diabetes mellitus. Pro Japan Acad $57: 381-384$

22. King AJ, Fernandes JR, Hollister-Lock J, Nienaber CE, BonnerWeir S, Weir GC (2007) Normal relationship of beta and nonbeta-cells not needed for successful islet transplantation. Diabetes 56:2312-2318

23. Stoves J, Lindley EJ, Barnfield MC, Burniston MT, Newstead CG (2002) MDRD equation estimates of glomerular filtration rate in potential living kidney donors and renal transplant recipients with impaired graft function. Nephrol Dial Transplant 17:2036-2037

24. Harnpicharnchai P, Jakovljevic J, Horsey E et al (2001) Composition and functional characterization of yeast $66 \mathrm{~S}$ ribosome assembly intermediates. Mol Cell 8:505-515

25. Kanwar YS, Sun L, Xie P, Liu FY, Chen S (2011) A glimpse of various pathogenetic mechanisms of diabetic nephropathy. Annu Rev Pathol 6:395-423

26. Lebreton A, Rousselle JC, Lenormand P et al (2008) 60S ribosomal subunit assembly dynamics defined by semiquantitative mass spectrometry of purified complexes. Nucleic Acids Res 36:4988-4999

27. Asahara S, Matsuda T, Kido Y, Kasuga M (2009) Increased ribosomal biogenesis induces pancreatic beta cell failure in mice model of type 2 diabetes. Biochem Biophys Res Commun 381:367-371

28. Mariappan MM, D'Silva K, Lee MJ et al (2011) Ribosomal biogenesis induction by high glucose requires activation of upstream binding factor in kidney glomerular epithelial cells. Am J Physiol Renal Physiol 300:F219-F230

29. Inagi R (2009) Endoplasmic reticulum stress in the kidney as a novel mediator of kidney injury. Nephron Exp Nephrol 112:e1-e9
30. Glassock RJ (2010) Is the presence of microalbuminuria a relevant marker of kidney disease? Curr Hypertens Rep 12:364-368

31. Matheson A, Willcox MD, Flanagan J, Walsh BJ (2010) Urinary biomarkers involved in type 2 diabetes: a review. Diabetes Metab Res Rev 26:150-171

32. Pucci L, Triscornia S, Lucchesi D et al (2007) Cystatin C and estimates of renal function: searching for a better measure of kidney function in diabetic patients. Clin Chem 53:480-488

33. Rigalleau V, Beauvieux MC, Le Moigne F et al (2008) Cystatin C improves the diagnosis and stratification of chronic kidney disease, and the estimation of glomerular filtration rate in diabetes. Diabetes Metab 34:482-489

34. Oddoze C, Morange S, Portugal H, Berland Y, Dussol B (2001) Cystatin $\mathrm{C}$ is not more sensitive than creatinine for detecting early renal impairment in patients with diabetes. Am J Kidney Dis 38:310-316

35. Jeon YK, Kim MR, Huh JE et al (2011) Cystatin C as an early biomarker of nephropathy in patients with type 2 diabetes. J Korean Med Sci 26:258-263

36. Kamijo-Ikemori A, Sugaya T, Yasuda T et al (2011) Clinical significance of urinary liver-type fatty acid-binding protein in diabetic nephropathy of type 2 diabetic patients. Diabetes Care 34:691-696

37. Okada H, Kikuta HT, Kobayashi T et al (2000) Connective tissue growth factor expressed in tubular epithelium plays a pivotal role in renal fibrogenesis. J Am Soc Nephrol 16:133-143

38. Nguyen TQ, Tarnow L, Jorsal A et al (2008) Plasma connective tissue growth factor is an independent predictor of end-stage renal disease and mortality in type 1 diabetic nephropathy. Diabetes Care 31:1177-1182 